\title{
Least Reliable Bits Coding (LRBC) for High Data Rate Satellite Communications
}

Mark Vanderaar and Paul Wagner

Sverdrup Technology, Inc.

Lewis Research Center Group

Brook Park, Ohio

and

James Budinger

National Aeronautics and Space Administration

Lewis Research Center

Cleveland, Ohio

Prepared for the

14th International Communications Satellite Systems Conference sponsored by the American Institute of Aeronautics and Astronautics Washington, D.C., March 22-26, 1992

\section{N/SA}




\section{LEAST RELIABLE BITS CODING (LRBC) FOR HIGH DATA RATE SATELLITE COMMUNICATIONS}

\author{
Mark Vanderaar* \\ Sverdrup Technology, Inc. \\ Lewis Research Center Group \\ Brook Park, Ohio 44142
}

\author{
James Budinger \\ National Aeronautics and Space \\ Administration \\ Lewis Research Center \\ Cleveland, Ohio 44135
}

\author{
Paul Wagner \\ Sverdrup Technology, Inc. \\ Lewis Research Center Group \\ Brook Park, Ohio 44142
}

\section{ABSTRACT}

An analysis and discussion of a bandwidth efficient multilevel / multi-stage [1] block coded modulation technique called Least Reliable Bits Coding (LRBC) is presented. LRBC uses simple multi-level component codes that provide increased error protection on increasingly unreliable modulated bits in order to maintain an overall high code rate that increases spectral efficiency. Further, soft-decision multi-stage decoding is used to make decisions on unprotected bits through corrections made on more protected bits.

Using analytical expressions and tight performance bounds it is shown that LRBC can achieve increased spectral efficiency and maintain equivalent or better power efficiency compared to that of Binary Phase Shift Keying (BPSK). Bit error rates (BER) vs. channel bit energy with Additive White Gaussian Noise (AWGN) are given for a set of LRB ReedSolomon (RS) encoded 8PSK modulation formats with an ensemble rate of $8 / 9$. All formats exhibit a spectral efficiency of $2.67=\left(\log _{2} 8\right)^{*} 8 / 9$ information bps/Hz. Bit by bit coded and uncoded error probabilities with soft-decision information are determined. These are traded with code rate to determine parameters that achieve good performance.

The relative simplicity of Galois field algebra vs. the Viterbi algorithm and the availability of high speed commercial VLSI for block codes indicates that LRBC using block codes is a desirable method for high data rate implementations.

\section{INTRODUCTION}

Future on-board processing communications satellites have been proposed using Frequency Division Multiple Access (FDMA) uplinks and Time Division Multiplexed (TDM) downlinks [2]. In some uplink cases, it will be found that accommodating a large number of FDM channels through a limited transponder or channel allocation bandwidth will require increased spectral efficiency. Bandwidth efficient modulation and coding techniques are necessary to achieve this efficiency without requiring an increase in signal power. In most downlink cases, the transmit power allocated for each high data rate TDM downlink beam will pose a more significant constraint on the system design than will the available spectrum allocation. Similar combined modulation and coding techniques optimized this time for power efficiency can satisfy this need. Further, attention must also be given to minimizing the complexity (weight, cost, and reliability) on-board the satellite and the complexity (cost) to the ground terminals. In an on-board processed FDMATDM system, a high rate multi-channel block decoder on the satellite would be an efficient method to protect hundreds or thousands of low to medium rate uplinks. Such a decoder

\footnotetext{
"Work Supported by Contract NAS3-252.66, Task Order 5601, Digital Systems Technology Development
}

would immediately follow a Multi-Channel Demultiplexer Demodulator (MCDD) that converts channelized carriers into demodulated time staggered data streams. A single, time shared encoder and modulator on board the satellite would enable power efficient TDM downlink processing in low cost ground terminals.

\section{ANALYSIS}

III.1 : 8PSK Error Regions

A meaningful performance measurement of $\mathrm{M}$-ary digital communications systems is the bit error probability in AWGN. The bit error probability is dependent on both the Euclidean distance properties of the signalling constellation and the Hamming distance of the bit to symbol mappings. A general equation that describes this relationship is

$$
P_{b}=\frac{1}{M \log _{2} M} \sum_{x=1}^{\left(\log _{2} M\right)} \sum_{j=0}^{M-1} \sum_{i=0}^{M-1}\left(b_{j x} \oplus b_{i x}\right) P_{i-j}
$$

where $M$ is the order of the $M$-ary scheme and $x$ is one of the $\log _{2} M$ bits being examined. The bjx's and $b_{i x}$ 's are the logical value (1 or 0 ) of the $x^{\text {th }}$ th bit in the ith or j'th decision region. The $P$ term represents the probability that decision region $i$ was received, given that decision region $\mathrm{j}$ was transmitted. These decision regions are illustrated in Figure 1 for 8PSK. The 8PSK decision region probabilities can be determined as sums of half-plane and quarter-plane probabilities written in terms of Q-functions. The Q-function definition and method of calculation is presented in Appendix A. A half-plane probability is given as [3]

$h_{\theta}=Q\left[d_{k} \sin \theta\right]$

where $d_{k}$ corresponds to the signal to noise ratio and $\theta$ is an angle defining the beginning of the half-plane. In the case of 8PSK, $d_{k}$ is the same for all signalling points and can be written as

$d_{k}=d_{\log _{2} M}=\sqrt{\frac{2\left(\log _{2} M\right) E_{b}}{N_{b}}}=\sqrt{\frac{6 E_{b}}{N_{b}}}$

where $E_{b}$ is the received bit energy and the zero mean white Gaussian noise has a two-sided power spectral density of $\mathrm{N}_{0} / 2$. A quarter-plane probability is the joint probability of two appropriate overlapping half-planes

$q_{\theta}=h_{\theta} h_{\theta+\frac{\pi}{2}}$

Using the above equations and the symmetry of the 8PSK constellation and the odd symmetry of the sine function, the decision region probabilities can be determined. For the case that the constellation point associated with the $A_{0}$ region 
was transmitted, $\mathrm{P}_{0}$ is the probability that no symbol error occurred and is written as

$P_{0}=1-P_{s}$

where $P_{S}$ is the symbol error probability as calculated in Appendix $\mathrm{B}$. To determine $\mathrm{P}_{1}$, the first quadrant probability $P_{0,1}$ is

$P_{0,1}=\left(1-Q\left[\sqrt{\frac{6 E_{b}}{N_{0}}} \sin \left(\frac{\pi}{8}\right)\right]\right)\left(1-Q\left[\sqrt{\frac{6 E_{b}}{N_{0}}} \sin \left(\frac{3 \pi}{8}\right)\right]\right)$

and $P_{1}$ is simply

$P_{1}=P_{0,1}-P_{0}$

Similar equations are used to determine the remaining decision region probabilities and are given as follows

$P_{1,2}=\left(Q\left[\sqrt{\frac{6 E_{b}}{N_{b}}} \sin \left(\frac{\pi}{8}\right)\right]\right)\left(1-Q\left[\sqrt{\frac{6 E_{b}}{N_{b}}} \sin \left(\frac{3 \pi}{8}\right)\right]\right)$

$P_{2}=P_{1,2}-P_{1}$

$P_{2,3}=\left(Q\left[\sqrt{\frac{6 E_{b}}{N_{b}}} \sin \left(\frac{3 \pi}{8}\right)\right]\right)\left(1-Q\left[\sqrt{\frac{6 E_{b}}{N_{b}}} \sin \left(\frac{\pi}{8}\right)\right]\right)$

$P_{3}=P_{2,3}-P_{2}$

$P_{3,4}=\left(Q\left[\sqrt{\frac{6 E_{b}}{N_{b}}} \sin \left(\frac{\pi}{8}\right)\right]\right)\left(Q\left[\sqrt{\frac{6 E_{b}}{N_{b}}} \sin \left(\frac{3 \pi}{8}\right)\right]\right)$

$P_{4}=P_{3,4}-P_{3}$

Alternatively, it is noticed that the probability of symbol error can also be written as the sum of two half-planes minus their overlapping area

$P_{s}=Q\left[\sqrt{\frac{6 E_{b}}{N_{0}}} \sin \left(\frac{\pi}{8}\right)\right]+Q\left[\sqrt{\frac{6 E_{b}}{N_{0}}} \sin \left(\frac{\pi}{8}\right)\right]-P_{4}$

and combining with the equation for Ps (from Appendix B) it is seen that

$\mathrm{P}_{4} \approx \mathrm{e}$

From the symmetry of the signalling constellation the following decision region probabilities immediately follow

$$
P_{5}=P_{3} \quad P_{6}=P_{2} \quad P_{7}=P_{1}
$$

Three symbol mappings defined in Table 1 are evaluated for their probability of bit error as a function of their decision regions. The following three expressions result

$P_{b N M}=\frac{1}{24}\left(28 P_{1}+24 P_{2}+36 P_{3}+8 P_{4}\right)$

$$
\begin{aligned}
& P_{b G C}=\frac{1}{24}\left(16 P_{1}+32 P_{2}+32 P_{3}+16 P_{4}\right) \\
& P_{b D P}=\frac{1}{24}\left(24 P_{1}+16 P_{2}+40 P_{3}+16 P_{4}\right)
\end{aligned}
$$

\begin{tabular}{|c|c|c|c|c|}
\hline $\begin{array}{c}\text { Symbol } \\
\text { Number }\end{array}$ & $\begin{array}{l}\text { Phase } \\
\text { Value }\end{array}$ & $\begin{array}{l}\text { Natural } \\
\text { Mapping }\end{array}$ & $\begin{array}{l}\text { Gray } \\
\text { Coding }\end{array}$ & $\begin{array}{l}\text { Decision } \\
\text { Parsing }\end{array}$ \\
\hline 0 & 0 & 000 & 000 & 000 \\
\hline 1 & $\pi / 4$ & 001 & 001 & 001 \\
\hline 2 & $\pi / 2$ & 010 & 011 & 010 \\
\hline 3 & $3 \pi / 4$ & 011 & 010 & 011 \\
\hline 4 & $\pi$ & 100 & 110 & 110 \\
\hline 5 & $5 \pi / 4$ & 101 & 111 & 111 \\
\hline 6 & $3 \pi / 2$ & 110 & 101 & 100 \\
\hline 7 & $7 \pi / 4$ & 111 & 100 & 101 \\
\hline
\end{tabular}

Table 1: 8PSK bit to symbol mappings

The BER vs. $E_{b} / N_{0}$ curves of these 8PSK mappings are shown in Figure 2. Due to the relative minimization of the dominating decision region probability $P_{1}$, gray coding exhibits the lowest $\mathrm{E}_{b} / \mathrm{N}_{0}$ to obtain a given BER. However, these equations do not indicate which bits are contributing to the weights on the decision regions. To accomplish this, $\mathrm{Pb}$ can be written in a matrix form that illustrates the individual bit contributions as follows

$P_{b}=\frac{1}{24}\left[\begin{array}{lll}W_{\text {Mib 0 }} & W_{\text {mib 0 }} & W_{\text {Irb 0 }} \\ W_{\text {Mib 1 }} & W_{\text {mib 1 }} & W_{\text {Irb 1 }} \\ W_{\text {Mib 2 }} & W_{\text {mib 2 }} & W_{\text {Irb 2 }} \\ W_{\text {Mib 3 }} & W_{\text {mib 3 }} & W_{\text {Irb 3 }} \\ W_{\text {Mib 4 }} & W_{\text {mib 4 }} & W_{\text {Irb 4 }}\end{array}\right]\left[\begin{array}{c}\text { Mrb } \\ \text { mrb } \\ \text { Irb }\end{array}\right]$

where the $W_{b d}$ 's are the number of ways in which a bit error $b$ can be caused by a decision region d multiplied by the probability of being in decision region $\mathrm{d}$. The Irb (least reliable bit), mrb (middle reliable bit), and Mrb (most reliable bit) represent the rightmost, middle, and leftmost bits in the bit to symbol mappings, respectively. Matrices for the three mappings studied are

$P_{b N M}=\frac{1}{24}\left[\begin{array}{ccc}0 P_{0} & o P_{0} & o P_{0} \\ 4 P_{1} & 8 P_{1} & 16 P_{1} \\ 8 P_{2} & 16 P_{2} & 0 P_{2} \\ 12 P_{3} & 8 P_{3} & 16 P_{3} \\ 8 P_{4} & 0 P_{4} & 0 P_{4}\end{array}\right]\left[\begin{array}{c}M r b \\ m r b \\ \mid r b\end{array}\right]$

$P_{b G C}=\frac{1}{24}\left[\begin{array}{ccc}o P_{0} & 0 P_{0} & 0 P_{0} \\ 4 P_{1} & 4 P_{1} & 8 P_{1} \\ 8 P_{2} & 8 P_{2} & 16 P_{2} \\ 12 P_{3} & 12 P_{3} & 8 P_{3} \\ 8 P_{4} & 8 P_{4} & 0 P_{4}\end{array}\right]\left[\begin{array}{c}M r b \\ m r b \\ 1 r b\end{array}\right]$ 
$P_{b D P}=\frac{1}{24}\left[\begin{array}{ccc}O P_{0} & O P_{0} & O P_{0} \\ 4 P_{1} & 4 P_{1} & 16 P_{1} \\ 8 P_{2} & 8 P_{2} & O P_{2} \\ 12 P_{3} & 12 P_{3} & 16 P_{3} \\ 8 P_{4} & 8 P_{4} & O P_{4}\end{array}\right]\left[\begin{array}{c}\mathrm{Mrb} \\ \mathrm{mrb} \\ \mathrm{Irb}\end{array}\right]$

Reading the columns, with special notice of the $\mathrm{P}_{1}$ terms that occupy the second row verifies that some bits are more likely to be in error than others. The less reliable bits result in the bulk of the errors. For example, Figure 3 shows the individual bit error rates of gray coded 8PSK where the Irb contributes more to the overall BER than the mrb and Mrb.

\section{III.2 : LRBC Based on Reed-Solomon Codes}

The remainder of this paper focuses on coding that takes advantage of the unequal bit by bit error probabilities. For this high data rate application, Reed-Solomon (RS) block codes are studied over convolutional codes because of the commercial availability of RS codec VLSI chips at data rates in the hundreds of megabits per second. This compares to Viterbi algorithm convolutional decoders available at tens of megabits per second. Also, as individual codewords are independent, block codes are well suited to time-shared decoding and independent burst decoding .

The character error correcting capability of the RS(n,k) in the absence error location information is

$\mathrm{t}=(\mathrm{n}-\mathrm{k}) / 2$

where $n$ is the total number of characters per codeword and $k$ is the number of information characters per codeword. The average channel character error rate $\mathrm{P}_{\mathrm{C}}$, is determined from the channel bit rate by

$P_{c}=1-\left(1-P_{b}\right)^{m}$

where $\mathrm{Pb}_{b}$ is the channel bit rate and $m=\log _{2}(n+1)$ is the number of bits in an RS character. An approximate expression for the decoded character error probability of a $t$ error correcting RS code is [4]

$P_{c d} \approx \sum_{i=t+1}^{n} \frac{i}{n}\left(P_{c}\right)^{i}\left(1-P_{c}\right)^{n-i}\left(\begin{array}{l}n \\ i\end{array}\right)$

and the resulting average decoded bit error probability is

$P_{b d}=\frac{2^{(m-1)} P_{c d}}{\left(2^{m}-1\right)}$

These expressions are used to determine the performance of the three 8PSK modulation schemes that are RS coded in a bit by bit fashion. In each case the ensemble code rate is $8 / 9, n=255$ and $k$ is shown in Table 2 .

\begin{tabular}{|l|c|r|r|r|r|}
\hline Bit & Case 1 & Case 2 & Case 3 & Case 4 & Case 5 \\
\hline $\mathrm{rb}$ & 171 & 199 & 207 & 213 & 227 \\
\hline $\mathrm{mrb}$ & NONE & 241 & 237 & 233 & 227 \\
\hline $\mathrm{Mrb}$ & NONE & 241 & 237 & 233 & 227 \\
\hline
\end{tabular}

Table $2: k$ Values for RS $(n, k)$
Figures 4, 5, 6 show the performance of the various coding schemes for each bit mapping. Figure 7 shows the best scheme from each mapping. Again, because of the minimization of the dominate $\mathrm{P}_{1}$ term, the best mapping uses gray coding.

The use of soft-decision information [5] can be used to further improve the performance of LRBC. When a coded bit correction is made, the received decision region is necessarilly in error. The likely symbol transmitted is the nearest one (in terms of Euclidean distance) with the corrected coded bit(s). Knowing this, the uncoded bits can be modified as necessary, resulting in a reduced probability of bit error.

To quantify the performance of LRBC with soft-decision information, further parsing of the decision regions is needed. This structure of sixteen decision regions is shown in Figure 8. To find the probabilities associated with each minor decision region, denoted $\mathrm{Pm}$, half and quarter-plane probabilities are again used. The following equations result

$\mathrm{Pm}_{0}=\mathrm{P}_{0} / 2$

$P m_{1}=\frac{1}{2}\left\{\left(1-Q\left[\sqrt{\frac{6 E_{b}}{N_{0}}} \sin \left(\frac{2 \pi}{8}\right)\right]\right)^{2}-P_{0}\right\}$

$P m_{2}=P_{1}-P m_{1}$

$P m_{3}=\frac{1}{2}\left\{\left(1-Q\left[\sqrt{\frac{6 E_{b}}{N_{0}}} \sin \left(\frac{4 \pi}{8}\right)\right]\right)^{2}-P_{0}-2 P_{1}\right\}$

$\mathrm{Pm}_{4}=\mathrm{P}_{2}-\mathrm{Pm}_{3}$

$P m_{5}=\left(1-Q\left[\sqrt{\frac{6 E_{b}}{N_{0}}} \sin \left(\frac{2 \pi}{8}\right)\right]\right)^{2}-P_{0}-P_{1}-P_{2}-P_{1}$

$P m_{6}=P_{3}-P m_{5}$

$\mathrm{Pm}_{7}=\mathrm{P}_{4} / 2$

$\mathrm{Pm}_{8}=\mathrm{Pm}_{7}$

$P m_{11}=P m_{4}$

$\mathrm{Pm}_{14}=\mathrm{Pm}_{1}$

$$
\begin{array}{ll}
\mathrm{Pm}_{9}=\mathrm{Pm}_{6} & \mathrm{Pm}_{10}=\mathrm{Pm}_{5} \\
\mathrm{Pm}_{12}=\mathrm{Pm}_{3} & \mathrm{Pm}_{13}=\mathrm{Pm}_{2}
\end{array}
$$

Three types of error events are considered to determine the effect of soft-decision information when only the lrb is coded. First, the probability that an uncoded mrb or Mrb is in error given a correction has been made on the coded Irb, called $\mathrm{Pb}_{\mathrm{b}} \mathrm{mrb}$ ci and $\mathrm{Pb} 1 \mathrm{Mrb}$ ci is calculated as

$$
\begin{aligned}
& P_{b x}=\frac{1}{M} \sum_{j=0}^{M-1}\left[\sum_{\substack{i=0 \\
i \text { even }}}^{2 M-1}\left(b_{j x} \oplus \frac{b i-2}{2} x\right)\left(b_{j} \operatorname{lr} b \oplus b_{-} \frac{i}{2} \operatorname{lr} b\right) P m_{n}+\right. \\
& \left.\sum_{i=0}^{2 M-1}\left(b_{j x} \oplus \frac{b}{2} x\right)\left(b_{j}\left|r b \oplus \frac{b}{2}\right| r b\right) P m_{n}\right] \\
& \text { i odd }
\end{aligned}
$$


where $\mathrm{i}-2 \mathrm{j}$ cannot equal 0 or 1 (not an error event) and the minor decision region index $\mathrm{n}$ is

$$
n=\begin{array}{ll}
i-2 j, & i-2 j \geq 0 \\
2 j-i-1, & i-2 j<0
\end{array}
$$

and thus

$P_{b 1 \text { mrb ci }}=P_{b \text { mrb }}\left(1-P_{d b} \mid r b\right)$

$P_{b 1}$ Mrb ci $=P_{b \text { Mrb }}\left(1-P_{d b} \mid r b\right)$

where $\mathrm{P}_{\mathrm{db}}$ Irb is the decoded bit error probability of the Irb. The second type of error event is the probability of bit error when no Irb correction or error has ocurred. This is written as

$P_{b x}=\frac{1}{M} \sum_{j=0}^{M-1} \sum_{i=0}^{M-1}\left(b_{j x} \oplus b_{i x}\right)\left(\overline{b_{j}\left|r b \oplus b_{i}\right| r b}\right) P|i-j|$

$P_{b 2} \mathrm{mrb} c i=P_{b} m r b$

$\mathrm{P}_{\mathrm{b} 2 \mathrm{Mrb} \mathrm{ci}}=\mathrm{P}_{\mathrm{b} \text { Mrb }}$

The third type of error event is the probability of bit error given a lrb decoding errror or erasure has occurred. This is evaluated as

$$
\begin{aligned}
& P_{b x}=\frac{1}{M} \sum_{j=0}^{M-1}\left[\sum_{\substack{i=0 \\
i \text { even }}}^{2 M-1}\left(b_{j x} \oplus \frac{b i-2}{2} x\right)\left(\overline{b_{j}\left|r b \oplus \frac{b}{2}\right| r b}\right) P m_{n}\right. \\
& \left.\sum_{\substack{i=0 \\
i \text { odd }}}^{2 M-1}\left(b_{j x} \oplus \frac{b}{\frac{i+1}{2}} x\right)\left(b_{j \text { Ir } b \oplus \frac{b_{i}}{2} \mid r b}\right) P m_{n}\right]
\end{aligned}
$$

again the minor decision region index $\mathrm{n}$ is

$$
\begin{array}{ll}
n=\begin{array}{ll}
i-2 j, & i-2 j \geq 0 \\
2 j-i-1, & i-2 j<0
\end{array} & \\
P_{b 3} \text { mr ci }=P_{b} \text { mrb } & P_{d b} \text { ib } \\
P_{b 3 \text { Mr ci }}=P_{b} \text { Mrb } & P_{d b} \mid r b
\end{array}
$$

where $x=m r b$ and Mrb. The probability that a mrb or Mrb are in error is then

$P_{b \text { mrb ci }}=P_{b 1} m b c i+P_{b 2 m r b i}+P_{b 3} m r c i$

$P_{b \text { Mrb ci }}=P_{b 1}$ Mrb ci $+P_{b 2}$ Mrb ci $+P_{b 3}$ Mrb ci

and overall probability of bit error is

$P_{b}=\frac{1}{3}\left(P_{b \mid r b}+P_{b m b c i}+P_{b}\right.$ Mrb ci $)$

This probability is plotted for the three mapping schemes using a $\mathrm{RS}(255,171)$ code on the Irb and channel correction information on the mrb and Mrb in Figure 9. This maintains the ensemble code rate of $8 / 9$. In this case gray coding is no longer the best mapping, in fact it is the worst. At higher
$\mathrm{E}_{\mathrm{b}} / \mathrm{N}_{\mathrm{O}}$ (greater than approximately $7 \mathrm{~dB}$ in the gray coded case and $8 \mathrm{~dB}$ in the other cases) the RS decoded Irb begins to exhibit very low probability of error. At this point the most likely error event when the mapping is gray coded is dependent on $\mathrm{Pm}_{3}$. In the other mappings, a $\mathrm{Pm}_{3}$ decision region results in an Irb correction and thus if necessary, mrb and Mrb corrections. The reason decision parsing is slightly better than a natural mapping has to do with the mapping of the mrb and Mrb. In decision parsing, the mrb and Mrb look like gray coded QPSK, so the most likely error event Pm4 will result in an error on either the mrb or Mrb, but not both. With a natural mapping, $\mathrm{Pm}_{4}$ is also the most likely error event, but can result in both a mrb and Mrb error.

For completeness, Figure 10 illustrates the effect of using shorter RS codes on the decision parsed mapping. Figure 11 illustrates a comparison between the best LRBC code with channel information, the best LRBC code without channel information, the best uncoded 8PSK scheme, point from a time domain simulation (with a union bound based extrapolated data point) of a rate $8 / 9$ convolutional coding system [6], and for comparison, uncoded BPSK scheme. Depending on the bit error rate a service class requires would dictate the code to be chosen.

The other issue is the hardware and computational complexity. The LRBC scheme with multi-stage decoding needs one RS codec operating at $1 / 3$ of the information data rate. Multi-stage decoding requires a buffer to wait for codeword decisions and a lookup table to make the corrections. Soft-decision quantization error is a factor, the number of quantization bits (and thus the size of the lookup table) is being studied. The LRBC without multi-stage decoding requires either one time-shared RS codec operating at the full data rate, or three parallel RS decoders each operating at $1 / 3$ the data rate. The rate difference in the codes will also require a degree of buffering. An LRBC scheme coding only the Irb and not using side information would require only one decoder at $1 / 3$ the data rate and some buffering. If the amount of coding gain was acceptable for the application, it would be the simplest to implement. The performance of a convolutional coding scheme plotted in Figure 11 is an existing implementation at 225 Mbps by COMSAT Laboratories [6]. It requires a set of 8 semi-custom ECL gate arrays to perform the add-compare-select function of the 16 state Viterbi decoder, a board of state memory, and hard decision logic. It is expected that the LRBC implementaion using commercially available VLSI technology will exhibit about a factor of 100 reduction in combined mass and power consumption.

\section{CONCLUSION AND FUTURE WORK}

An analysis of the performance of LRBC has been presented. It is shown that a rate $8 / 9$, spectrally efficient 2 information bps $/ \mathrm{Hz}$ LRBC can improve power efficiency over BPSK. Comparisons were presented on the different approaches to LRBC in terms of performance and complexity. An existing equivalent convolutional coding scheme was also used for comparison.

In the future, other types of block codes will be compared to the performance of the RS codes. The use of concatenated coding schemes will also be investigated. Optimization for power efficient applications such as high rate return links 
from the space exploration initiative program will be addressed.

Presently, a LRBC hardware prototype is being developed at NASA Lewis Research Center. It will be integrated into a programmable modem developed by COMSAT Laboratories for NASA Lewis [7]. The LRBC hardware will be compared to COMSAT's Coded Trellis Modulation (CTM) hardware in a laboratory environment.

\section{APPENDIX A}

$Q(x)$ is defined as

$Q(x)=\frac{1}{2} \operatorname{erfc}\left(\frac{x}{\sqrt{2}}\right)$

For the 8PSK case $\mathrm{x}$ is defined as

$x=\sqrt{\frac{3 E_{0}}{N_{0}}} \sin \left(\frac{\pi}{8}\right)$

The erfc $(x)$ can be written in series notation as

$\operatorname{erfc}(x)=1-\frac{2}{\sqrt{\pi}}\left(x-\frac{x^{3}}{3 \cdot 1 !}+\frac{x^{5}}{5 \cdot 2 !}-\frac{x^{7}}{7 \cdot 3 !}+\right)$

and thus

$$
\begin{array}{r}
Q(x)=\frac{1}{2}-\frac{1}{\sqrt{\pi}}\left([x / \sqrt{2}]-\frac{[x / \sqrt{2}]^{3}}{3 \cdot 1 !}+\right. \\
\left.\qquad \frac{[x / \sqrt{2}]^{5}}{5 \cdot 2 !}-\frac{[x / \sqrt{2}]^{7}}{7 \cdot 3 !}+\cdots\right)
\end{array}
$$

in series notation this reduces to

$Q(x)=\frac{1}{2}-\frac{1}{\sqrt{2 \pi}} \sum_{k=0}^{\infty}\left(\frac{(-1)^{k} x^{(2 k+1)}}{2^{k}(2 k+1) \cdot k !}\right)$

As $\mathrm{k}$ becomes large, the direct evaluation of this alternating series becomes more inaccurate. Thus, the terms of $Q(x)$ are found by the ratio of the $k$ and $k-1$ terms. This ratio is written as

$\frac{\operatorname{term}_{k}}{\operatorname{term}_{k-1}}=\frac{\left(\frac{(-1)^{k} x^{2 k}+1}{2^{k}(2 k+1) \cdot k !}\right)}{\left(\frac{(-1)^{k-1} x^{2(k-1)+1}}{2^{k-1}(2(k-1)+1) \cdot(k-1) !}\right)}=\frac{x^{2}(2 k-1)}{2(2 k+1) \cdot k}$

\section{APPENDIX B}

A tight bound on the probability of symbol error for 8PSK is written as [8]

$P_{s} \approx 2 Q\left[\sqrt{\frac{6 E_{b}}{N_{0}}} \sin \left(\frac{\pi}{8}\right)\right]-e$

The error term e that results in an upper bound for $P_{S}$ is defined as $\theta=\frac{\tan \left(\frac{\pi}{8}\right)}{2 \pi}\left[E_{i}(y)-\left(\frac{\frac{\exp ^{-y}}{y}-E_{i}(y)}{2 \cos ^{2}\left(\frac{\pi}{8}\right)}\right)\right]$

where $y$ is

$y=\frac{3 E_{b}}{N_{0}}$

and $E_{i}(y)$ is the exponential integral defined in [9] as the series

$E_{i}(y)=-\gamma-\ln (y)+\sum_{k=1}^{\infty} \frac{y^{k}}{k \cdot k !}$

where $\gamma$ is Euler's constant. The ratio of the $k$ to $k-1$ terms is

$\frac{\operatorname{term}_{k}}{\operatorname{term}_{k-1}}=\frac{\left(\frac{y^{k}}{k \cdot k !}\right)}{\left(\frac{y^{k-1}}{(k-1) \cdot(k-1) !}\right)}=\frac{y(k-1)}{k^{2}}$

\section{REFERENCES}

[1] Imai, H.; Hirakawa, S. : "A New Multilevel Coding Method Using Error-Correcting Codes", IEEE Trans. Comm., vol. 23, no. 3, pp. 371-377, May 1977.

[2] Harrold, J. L.; Budinger, J. M.; and Stevens,G. H.: OnBoard Switching and Processing, Proc. IEEE, vol. 78, No. 7, pp.1206-1212, July 1990.

[3] M. I. Irshid and I. S. Salous, "Bit Error Probability for Coherent M-ary PSK Systems", IEEE Trans. Comm., vol. 39, No. 3, pp. 349-352, March 1991.

[4] B. Sklar, "Digital Communications Fundamentals and Applications", Prentice Hall, pp. 306, 1988.

[5] D. Hatzipapafotiou, "Codulation Study Final Report", to General Electric Company Military and Data Systems Operations, January 1989.

[6] COMSAT Laboratories, "Final Report : Advanced Modulation Technology Development for Earth Station Demodulator Applications", submitted to NASA Lewis Research Center under NASA contract no. NAS3-24680.

[7] Poklemba, J.: "Programmable Digital Modem", Proceedings of the Space Communications Technology Conference : Onboard Processing and Switching, NASA Conference Publication 3132, pp. 263-271, November 1991.

[8] C. M. Chie, "Bounds and Approximations for Rapid Evaluation of Coherent MPSK Error Probabilities", IEEE Trans. Comm., vol. COM-33, No. 3, pp. 271-273, March 1985.

[9] M. Spiegel, "Mathematical Handbook of Formulas and Tables", McGraw-Hill Book Company, pp. 183, 1968. 


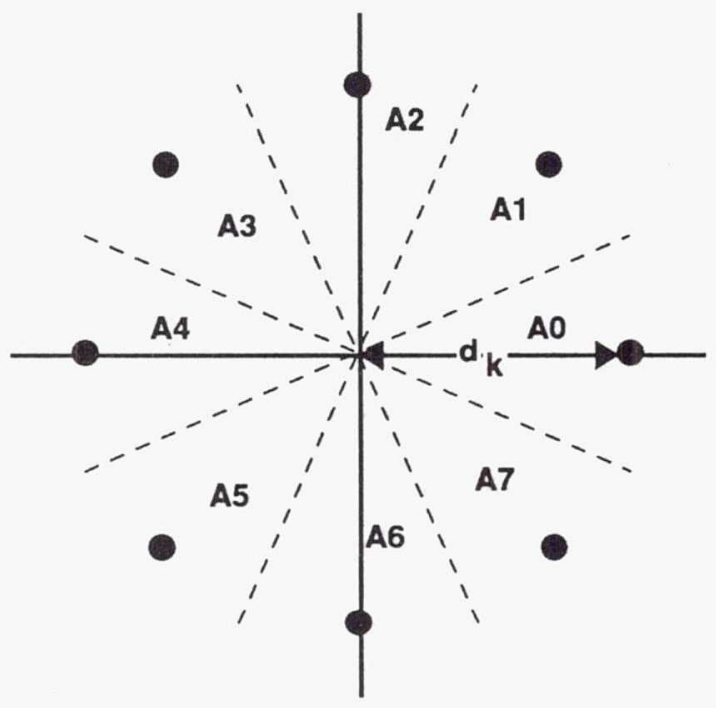

Figure 1: 8PSK Decision Regions

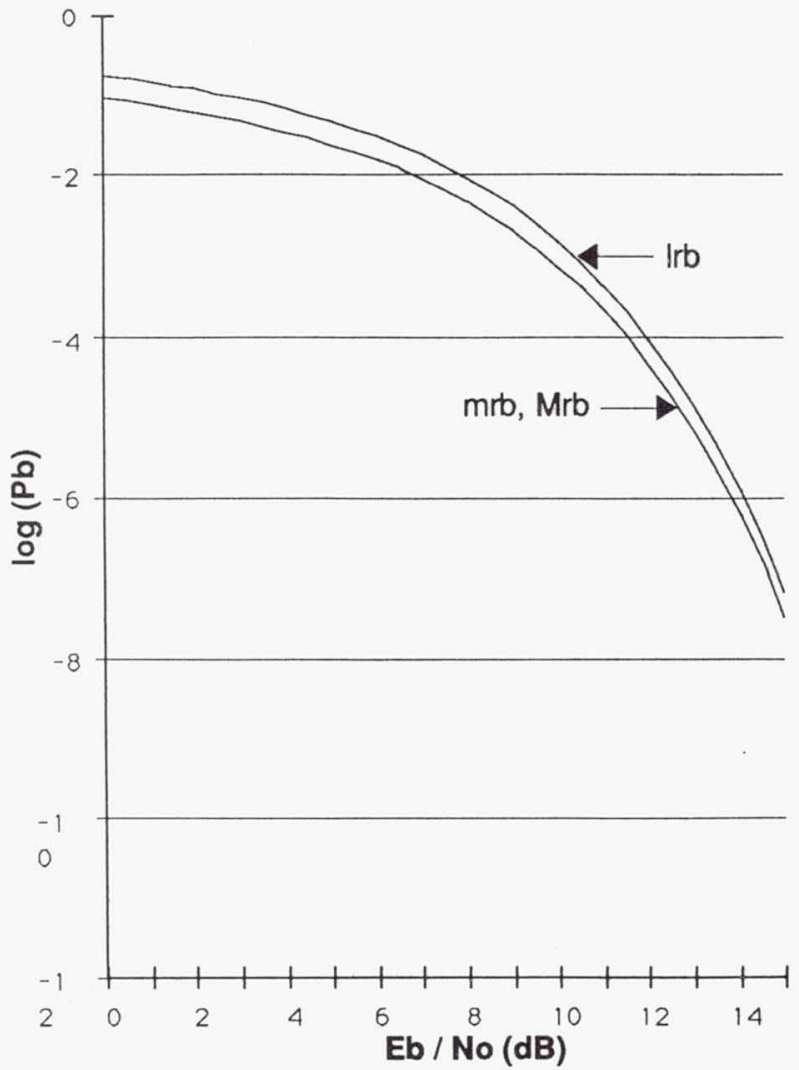

Figure 3: Gray Coding Bit by Bit Error Probabilities

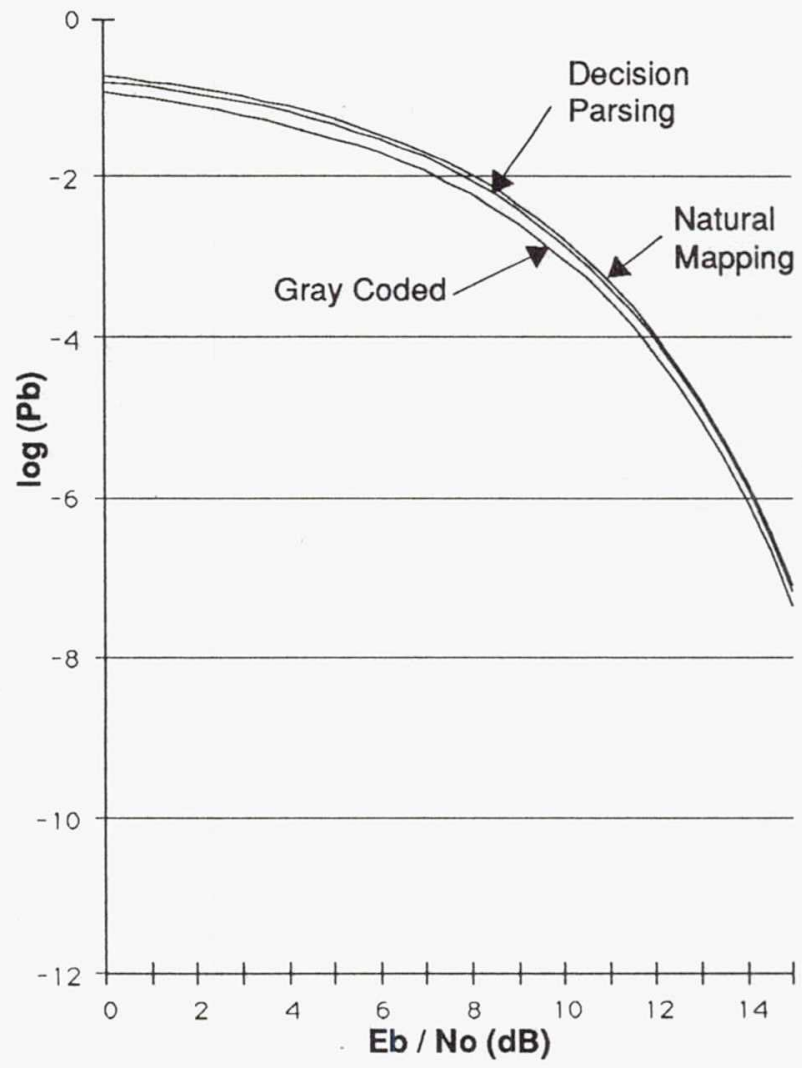

Figure 2: Uncoded 8PSK Comparison

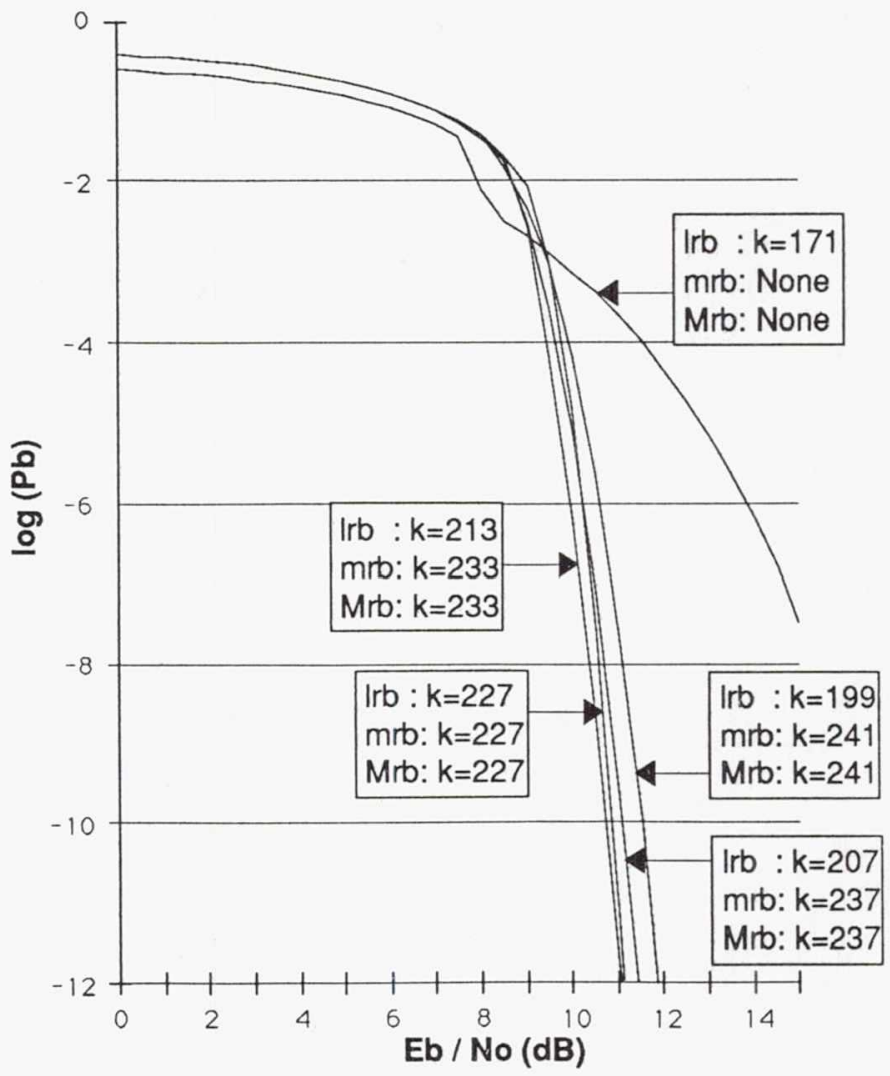

Figure 4 : LRBC with Natural Mapping 


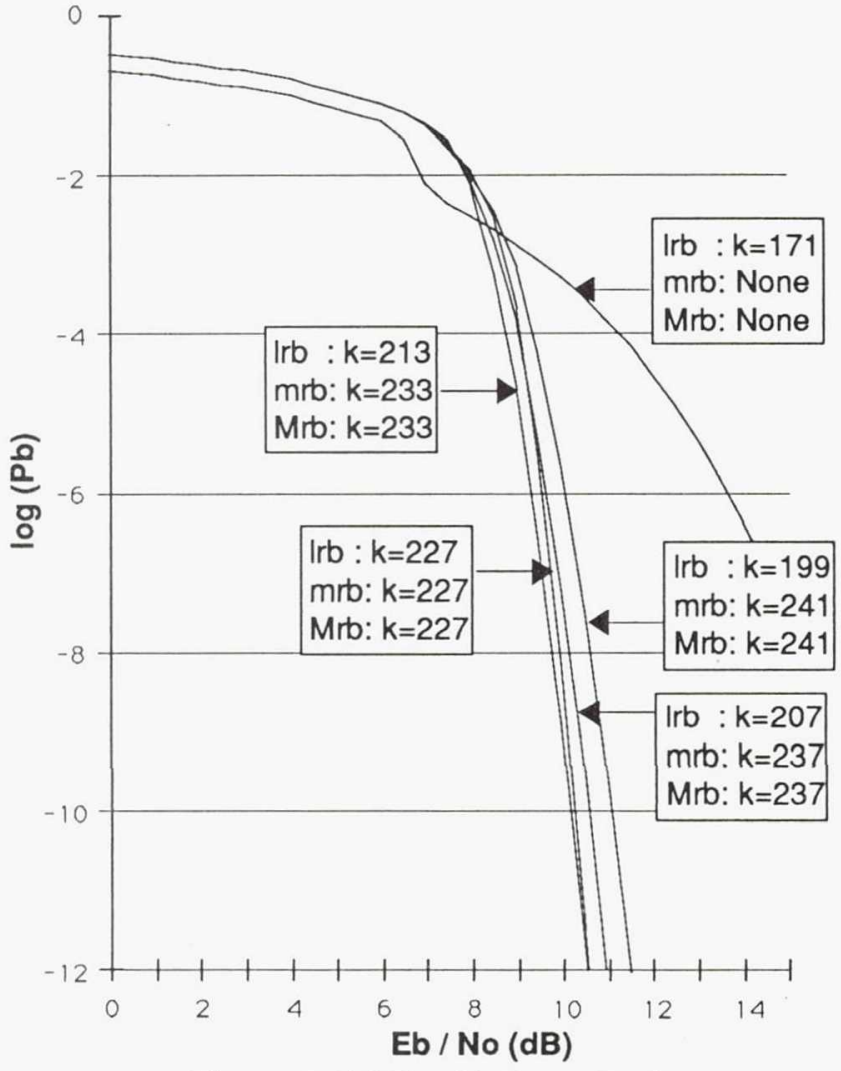

Figure 5: LRBC with Gray Coding

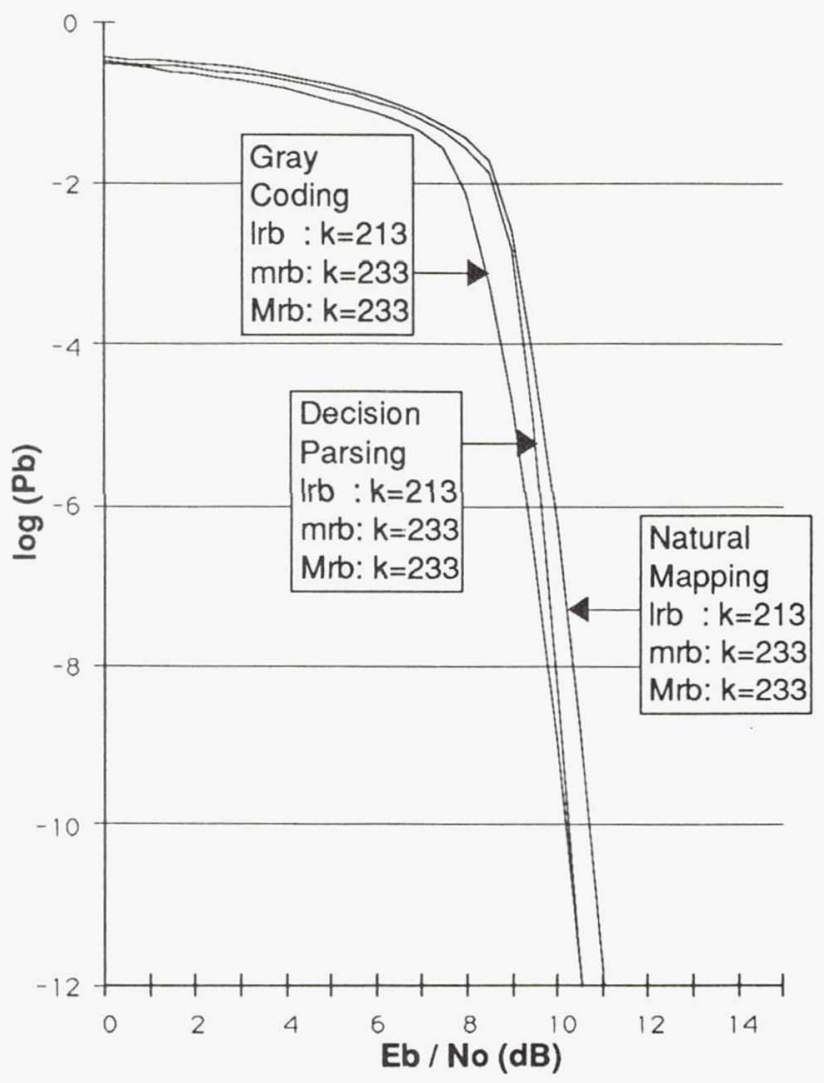

Figure 7: LRBC Comparison

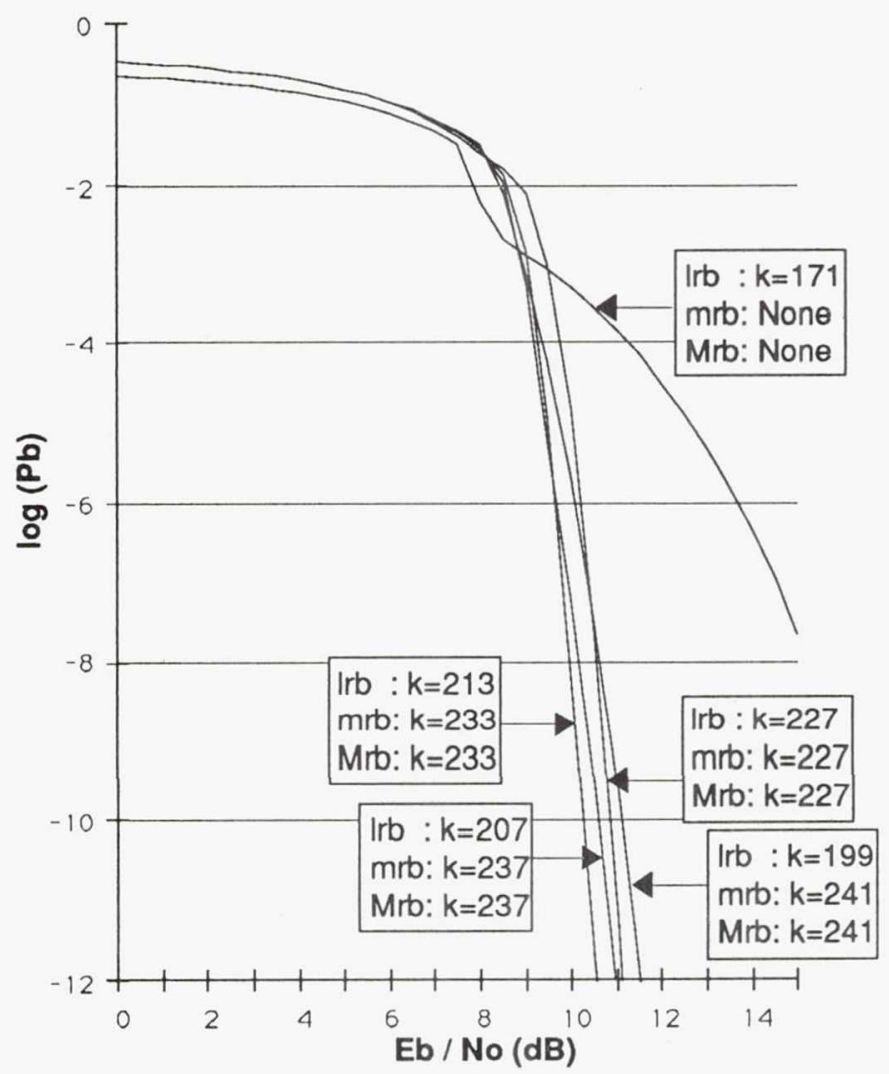

Figure 6: LRBC with Decision Parsing

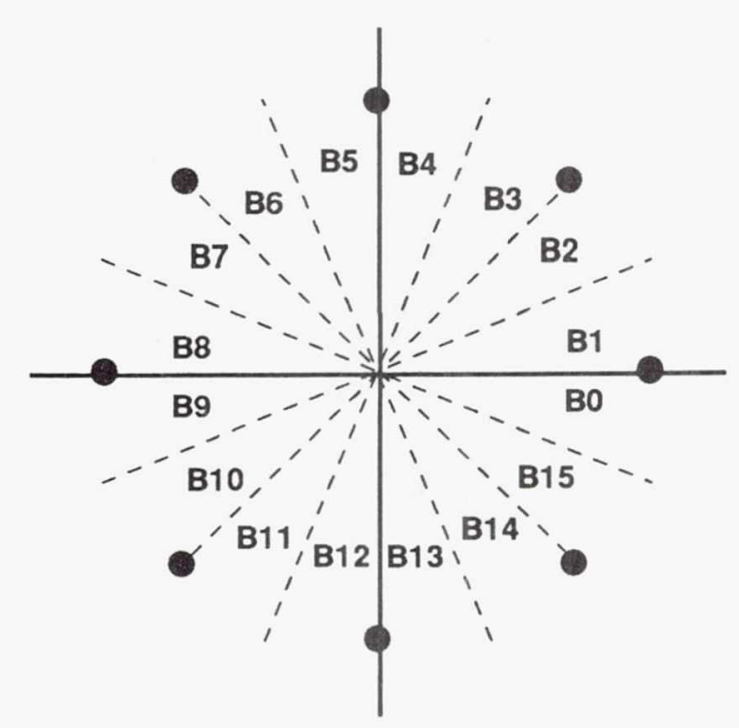

Figure 8: Minor Decision Regions 


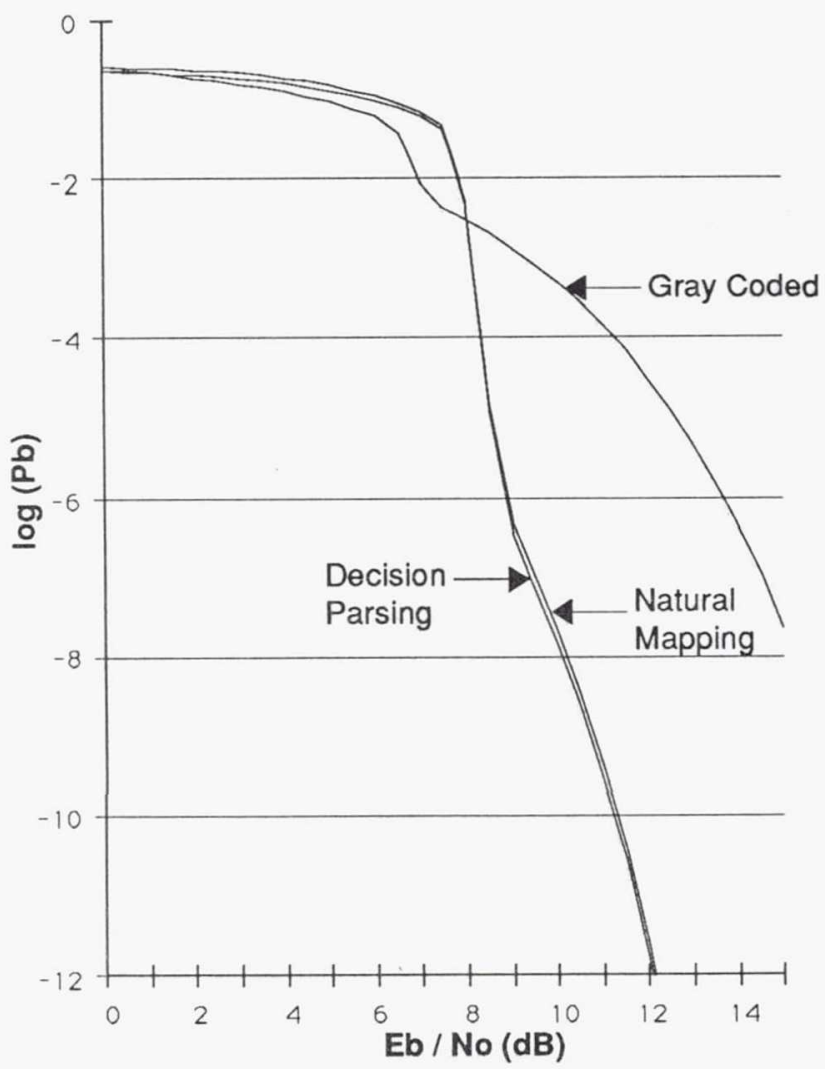

Figure 9: Multi-Stage LRBC

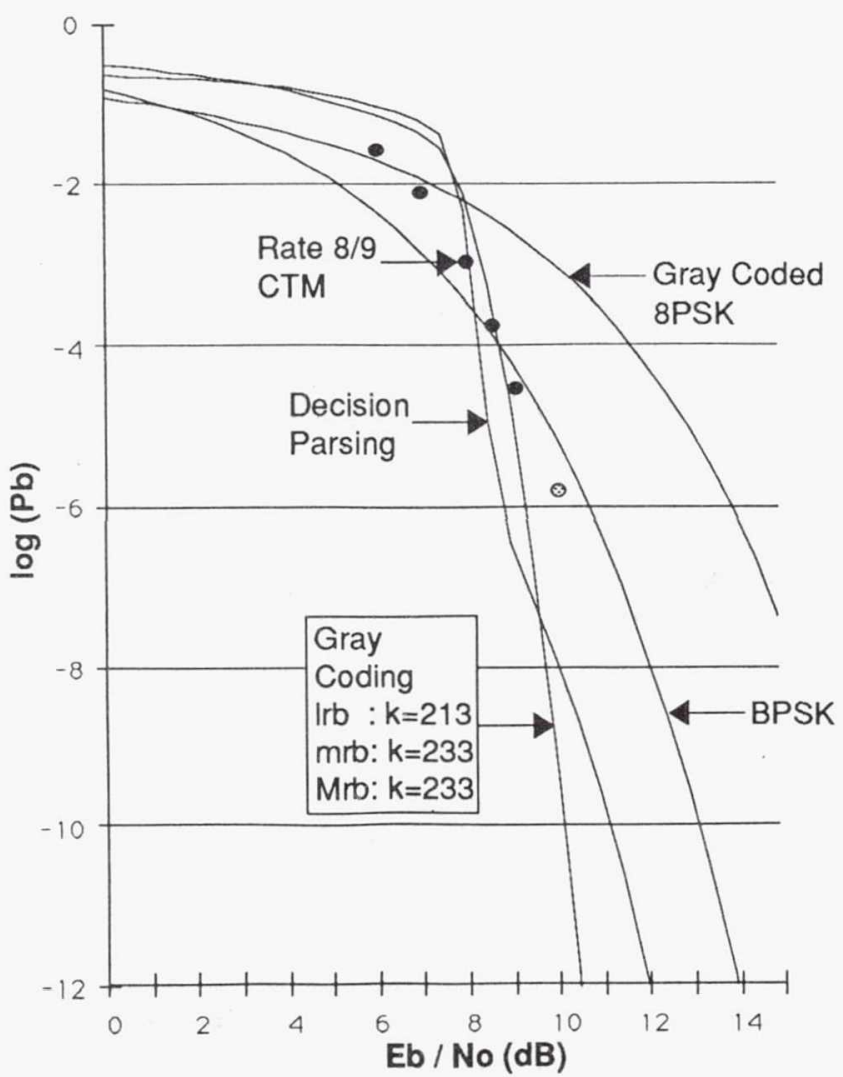

Figure 11: Overall Comparison

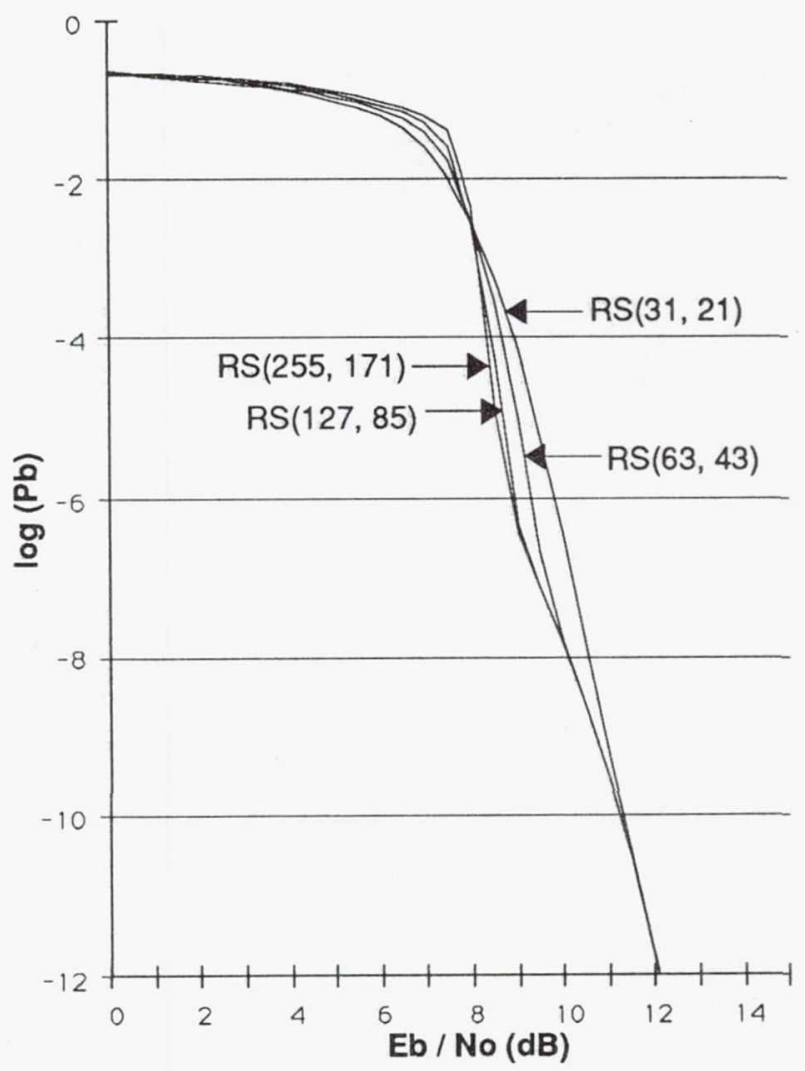

Figure 10: Multi-Stage Decision Mapped LRBC 
Public reporting burden for this collection of information is estimated to average 1 hour per response, including the time for reviewing instructions, searching existing data sources, gathering and maintaining the data needed, and completing and reviewing the collection of information. Send comments regarding this burden estimate or any other aspect of this collection of information, including suggestions for reducing this burden, to Washington Headquarters Services, Directorate for information Operations and Reports, 1215 Jefferson Davis Highway, Suite 1204, Arlington, VA 22202-4302, and to the Office of Management and Budget, Paperwork Reduction Project (0704-0188), Washington, DC 20503.

\begin{tabular}{|l|c|c|}
\hline 1. AGENCY USE ONLY (Leave blank) & $\begin{array}{c}\text { 2. REPORT DATE } \\
1992\end{array}$ & $\begin{array}{r}\text { 3. REPORT TYPE AND DATES COVERED } \\
\text { Technical Memorandum }\end{array}$ \\
\hline
\end{tabular}

\section{TITLE AND SUBTITLE}

Least Reliable Bits Coding (LRBC) for High Data

Rate Satellite Communications

6. AUTHOR(S)

Mark Vanderaar, Paul Wagner, and James Budinger

7. PERFORMING ORGANIZATION NAME(S) AND ADDRESS(ES)

National Aeronautics and Space Administration

Lewis Research Center

Cleveland, Ohio 44135-3191

9. SPONSORING/MONITORING AGENCY NAMES(S) AND ADDRESS(ES)

National Aeronautics and Space Administration

Washington, D.C. 20546-0001

\section{FUNDING NUMBERS}

WU $-506-72$
8. PERFORMING ORGANIZATION REPORT NUMBER

E -6848

11. SUPPLEMENTARY NOTES

Mark Vanderaar and Paul Wagner, Sverdrup Technology, Inc., Lewis Research Center Group, 2001 Aerospace Parkway, Brook Park, Ohio 44142 (work funded by NASA Contract NAS3-25266). James Budinger, NASA Lewis Research Center. Responsible person, James Budinger, (216) 433-8656.

12a. DISTRIBUTION/AVAILABILITY STATEMENT

12b. DISTRIBUTION CODE

Unclassified - Unlimited

Subject Category 32

13. ABSTRACT (Maximum 200 words)

An analysis and discussion of a bandwidth efficient multi-level/multi-stage block coded modulation technique called Least Reliable Bits Coding (LRBC) is presented. LRBC uses simple multi-level component codes that provide increased error protection on increasingly unreliable modulated bits in order to maintain an overall high code rate that increases spectral efficiency. Further, soft-decision multi-stage decoding is used to make decisions on unprotected bits through corrections made on more protected bits. Using analytical expressions and tight performance bounds it is shown that LRBC can achieve increased spectral efficiency and maintain equivalent or better power efficiency compared to that of Binary Phase Shift Keying (BPSK). Bit error rates (BER) vs. channel bit energy with Additive White Gaussian Noise (AWGN) are given for a set of LRB Reed-Solomon (RS) encoded 8PSK modulation formats with an ensemble rate of $8 / 9$. All formats exhibit a spectral efficiency of $2.67=\left(\log _{2} 8\right) * 8 / 9$ information bps/Hz. Bit by bit coded and uncoded error probabilities with soft-decision information are determined. These are traded with code rate to determine parameters that achieve good performance. The relative simplicity of Galois field algebra vs. the Viterbi algorithm and the availability of high speed commercial VLSI for block codes indicates that LRBC using block codes is a desirable method for high data rate implementations.

14. SUBJECT TERMS

Satellite; Communication; Modulation and coding; Spectral efficiency 15. NUMBER OF PAGES 10 16. PRICE CODE $\mathrm{AO} 2$

17. SECURITY CLASSIFICATION OF REPORT

Unclassified
18. SECURITY CLASSIFICATION OF THIS PAGE

Unclassified
19. SECURITY CLASSIFICATION OF ABSTRACT Unclassified 